Proyecciones

Vol. 20, No 1, pp. 65-82, May 2001.

Universidad Católica del Norte

Antofagasta - Chile

\title{
AN ASYMPTOTIC FORMULA FOR THE NUMBER OF EIGENVALUES OF A DIFFERENTIAL OPERATOR
}

\author{
E. ADIGUZELOV, H. AVCI and E. GUL \\ Yildiz Technical University - TURKEY
}

\begin{abstract}
In this work, it is proved that the spectrum of an differential operator with unbounded operator coefficients in elliptic type with partial derivatives is pure discrete and an asymptotic formula is found for the number of eigenvalues of this operator.
\end{abstract}




\section{Introduction}

The asymptotic behavior of the spectrum of Sturm-Liouville operator with operator coefficient had been firstly studied by A.G. Kostyuchenko and B.M. Levitan, [1]. Some works about this subject had been done later by M.L. Gorbacuk, [2], V.. Gorbacuk and M.L. Gorbacuk, [3], [4], M. Otelbayev ,[5], M.Z. Solomyak ,[6], F.G. Maksudov, M. Bairamoglu and E.E. Adiguzelov, [7], and etc. In this work, the spectrum of self adjoint operator $L$ forming with differential expression

$l(u)=-\sum_{i, j=1}^{n} \frac{\partial}{\partial x_{i}}\left(a_{i j}(x) \frac{\partial u}{\partial x_{i}}\right)+Q(x) u, \quad\left(x=\left(x_{1}, x_{2}, \ldots, x_{n}\right) \in \mathbb{R}^{n}\right)$

defined in $H_{1}=L_{2}\left(H ; \mathbb{R}^{n}\right)$ is studied and an asymptotic formula for the number of eigenvalues of $L$ is found, where $H$ denotes the Hilbert space. Here, we will accept that the coefficients $a_{i j}(x) \quad(i, j=$ $1, \ldots, n)$ and $Q(x)$ in (1.1) satisfy the following conditions:

1) Real valued functions $a_{i j}(x)=a_{j i}(x)$ have bounded derivatives $\frac{\partial a_{i j}(x)}{\partial x_{k}}(i, j, k=1, \ldots, n)$ in $\mathbb{R}^{n}$.

2) There are positive numbers $\nu$ and $\mu$ such that

$$
\nu \sum_{i=1}^{n} \xi_{i}^{2} \leq \sum_{i, j=1}^{n} a_{i j}(x) \xi_{i} \xi_{j} \leq \mu \Sigma_{i=1}^{n} \xi_{i}^{2}
$$

3) $Q(x): D(Q(x)) \rightarrow H$ is a self adjoint operator, where $D(Q(x)) \subset$ $H$ for each $x \in \mathbb{R}^{n}$. The set $D=\cap_{x \in \mathbb{R}^{n}} D(Q(x))$ is dense in $H$. $Q(x) \geq I$ and $Q^{-1}(x) \in \sigma_{\infty}(H)$.

4) There are constants $0<a<\frac{3}{2}$ and $B>0$ such that

$$
\left\|[Q(x)-Q(\xi)] Q^{-a}(x)\right\| \leq B|x-\xi|
$$

for $|x-\xi| \leq 1$

5) There is a constant $l>0$ satisfying $Q^{-l}(x) \in \sigma_{1}(H)$ and $\int_{\mathbb{R}^{n}}\left\|Q^{-l}(x)\right\|_{1} d x<\infty$ for $x \in \mathbb{R}^{n}$.

6) There is a positive valued function $f$ satisfying

$$
\left\|e^{-c t Q(\xi)}\right\|_{1} \leq\left\|e^{-f(c) t Q(x)}\right\|_{1}
$$


for $c>0, t>0$ and $|x-\xi| \leq 1$.

7) For every $M>0$

$$
\int_{\mathbb{R}^{n}} t r e^{-M t Q(x)} d x=O(1) \int_{\mathbb{R}^{n}} t r e^{-t Q(x)} d x .
$$

Here, $\operatorname{tr} A=\operatorname{trace} A$ is the sum of the eigenvalues of a kernel operator A.

8) Let $\alpha_{1}(x) \leq \alpha_{2}(x) \leq \ldots \leq \alpha_{m}(x) \leq \ldots$ be eigenvalues of operator $Q(x)$. Assume that the functions $\alpha_{1}(x), \alpha_{2}(x), \ldots$ are measurable functions. Let

$$
\begin{gathered}
\rho(\lambda)=\Sigma_{i} \int_{\alpha_{i}(x)<\lambda} \phi(x)\left(\lambda-\alpha_{i}(x)\right)^{\frac{n}{2}} d x, \\
\phi(x)=\int_{\mathbb{R}^{n}} e^{-\Sigma_{i, j=1}^{n} a_{i j}(x) \xi_{i} \xi_{j}} d \xi, \quad\left(\xi=\left(\xi_{1}, \ldots, \xi_{n}\right)\right) .
\end{gathered}
$$

Suppose that there is a constant $a_{0}>0$ such that

$$
\lambda \rho^{\prime}(\lambda) \leq a_{0} \rho(\lambda)
$$

for positive big values of $\lambda$. Let $D^{\prime}$ denotes the set of elements in the form $\sum_{k=1}^{m} \varphi_{k}(x) f_{k}$ of the space $H_{1}$, where $\varphi_{1}(x), \varphi_{2}(x), \ldots, \varphi_{m}(x)$, are scaler functions having compact support and continuous derivatives of second order defined in $\mathbb{R}^{n}$ and $\left\{f_{k}\right\}_{k=1}^{m} \subset D$. It is known that $\overline{D^{\prime}}=H_{1}$. The linear operator $L^{\prime}$ defined in the form

$$
L^{\prime} u=l(u)=-\sum_{i, j=1}^{n} \frac{\partial}{\partial x_{i}}\left(a_{i j}(x) \frac{\partial u}{\partial x_{j}}\right)+Q(x) u, \quad u \in D^{\prime}
$$

is a symmetric and positive operator in $H_{1}$. Suppose that the operator $L$ which is closure of $L^{\prime}$ is pure discrete. Let

$$
\begin{aligned}
& L_{0}\left(\eta, \frac{\partial}{\partial x}\right)=-\sum_{i, j=1}^{n} a_{i} j(\eta) \frac{\partial^{2}}{\partial x_{i} \partial x_{j}} \\
& L_{1}\left(x, \frac{\partial}{\partial x}\right)=-\sum_{i, j=1}^{n} \frac{\partial a_{i} j(x)}{\partial x_{i}} \frac{\partial}{\partial x_{j}} .
\end{aligned}
$$

To solve our main problem, we shall firstly study the Green function of Cauchy problem in the following: 


$$
\left\{\begin{array}{c}
\frac{\partial u}{\partial t}=-L u=-\left[L_{0}\left(x, \frac{\partial}{\partial x}\right)+L_{1}\left(x, \frac{\partial}{\partial x}\right)+Q(x)\right] u \\
u(x, 0)=\psi(x) ; \quad u=u(x, t), \quad \psi(x) \in L_{2}\left(H, \mathbb{R}^{n}\right)
\end{array}\right.
$$

We shall look this for the Green function given by the form

$$
\begin{aligned}
& G(x, y, t)=G_{1}(x-y, y, t) e^{-t Q(y)}+ \\
& \int_{0}^{t} d \tau \int_{\mathbb{R}^{n}} G_{1}(x-\xi, \xi, t-\tau) e^{-(t-\tau) Q(\xi)} \varphi(\xi, y, \tau) d \xi
\end{aligned}
$$

We will use parametric method for this. Here $G_{1}(x-y, y, t)$ is a function of the form

$$
G_{1}(x-y, y, t)=R(x-y) G_{0}(x-y, y, t),
$$

which has continuous derivatives of second order, where

$$
R(x)=\left\{\begin{array}{ll}
1 & |x| \leq \frac{1}{2} \\
0 & |x|>1
\end{array}(1.5)\right.
$$

And $G_{0}(x-y, \eta, t)$ denotes the Green function of the problem

$$
\left\{\begin{array}{c}
\frac{\partial u}{\partial t}=-L_{0}\left(\eta, \frac{\partial}{\partial x}\right) u \\
u(x, 0)=\psi(x) ; \quad \psi(x) \in L_{2}\left(H, \mathbb{R}^{n}\right)
\end{array}\right.
$$

The function $\varphi(x, y, t)$ in (1.3) is an unknown operator function that it must be here found. If the equality

$$
\frac{\partial G}{\partial t}=-L G=-\left[L_{0}\left(x, \frac{\partial}{\partial x}\right)+L_{1}\left(x, \frac{\partial}{\partial x}\right)+Q(x)\right] G
$$

is true, then we can take $G(x, y, t)$ as a Green function of problem (1.2). And so, the integral equation

$$
\varphi(x, y, t)=K(x, y, t)+\int_{0}^{t} d \tau \int_{\mathbb{R}^{n}} K(x, \xi, t-\tau) \varphi(\xi, y, \tau) d \xi
$$


is obtained, where

$$
\begin{gathered}
K(x, y, t)=\quad-e^{-t Q(y)}\left[\frac{\partial}{\partial t}+L_{0}\left(x, \frac{\partial}{\partial x}\right)\right] G_{1}(x-y, y, t)+ \\
+[Q(y)-Q(x)] e^{-t Q(y)} G_{1}(x-y, y, t)- \\
-e^{-t Q(y)} L_{1}\left(x, \frac{\partial}{\partial x}\right) G_{1}(x-y, y, t) .
\end{gathered}
$$

\section{Preliminaries}

Now, before we give main results, we remind some of its definitions used in this paper as below:

\section{About Measurability}

Let $f(x)$ and $\left\{f_{i}(x)\right\}_{i=1}^{\infty}$ be any functions defined on Euclidean space $\mathbb{R}^{n}$ that their values belong to a separable Hilbert space $H$.

Definition 2.1 A function defined on $\mathbb{R}^{n}$ whose range is a countable set, is said to be countable valued if it takes on a measurable set its all values except zero.

Definition 2.2 A function $f(x)$ is said to be strong measurable if there exists a sequence $\left\{f_{i}(x)\right\}_{i=1}^{\infty}$ of countable valued functions such that $\lim _{i \rightarrow \infty}\left\|f_{i}(x)-f(x)\right\|_{H}=0$ in almost every where on $\mathbb{R}^{n}$.

The space $H_{1}=L_{2}\left(H ; \mathbb{R}^{n}\right)$

Let us denote with $H_{1}=L_{2}\left(H ; \mathbb{R}^{n}\right)$ the set of strong measurable functions $f$ of satisfying the condition

$$
\int_{\mathbb{R}^{n}}\|f(x)\|_{H}^{2} d x<\infty \quad x=\left(x_{1}, x_{2}, \ldots, x_{n}\right) \in \mathbb{R}^{n}
$$

If the inner product of two element $f$ and $g$ of the set $H_{1}$ is defined by the formula

$$
(f, g)=\int_{\mathbb{R}^{n}}(f(x), g(x))_{H} d x
$$

then the set $H_{1}$ forms a separable Hilbert space, [8].

The spaces $\sigma_{\infty}(H)$ and $\sigma_{1}(H)$

The set of all complete continuous operators from $H$ to $H$ is denoted with $\sigma_{\infty}$. Let $A \in \sigma_{\infty}(H)$ be an operator which is different from zero and $A^{*}$ be adjoint operator of $A$. Let $s_{1} \geq s_{2} \geq \ldots \geq s_{i} \geq \ldots$ be the eigenvalues which is different from zero of positive self-adjoint operator 
$\left(A^{*} A\right)^{1 / 2}$. Here every eigenvalues is repeated according to multiplicity number. Moreover $, s_{1}, s_{2}, \ldots, s_{i}, \ldots$ are the positive numbers. These are called the $s$-numbers of operator $A$. We can write the $s$-numbers of $A$ in the form $s_{i}(A)$ and show the number of the $s$-numbers of $A$ with $\nu(A)$. Not that $\nu(A)$ can be finite or infinite. We shall denote the union of the operator " 0 " and the set of all operators $A \in \sigma_{\infty}(H)$ satisfying

$$
\sum_{i=1}^{\nu(A)} s_{i}(A)<\infty
$$

with $\sigma_{1}$ or $\sigma_{1}(H) . \sigma_{1}$ is a Banach space, [9]. In this space, every operator $A \neq 0$ is defined by

$$
\|A\|_{\sigma_{1}(H)}=\sum_{i=1}^{\nu(A)} s_{i}(A)
$$

and we accept that $\|0\|_{\sigma_{1}(H)}=0,[9]$.

Along this study, we shall denote positive constants with $c$ which may not be same and the norm of the kernel operator from $H$ to $H$ in space $\sigma_{1}(H)$ with $\|\cdot\|_{1}$ as in [9]. Moreover, here we shall use the inequalities

$$
\begin{aligned}
& \|A B\|_{1} \leq\|A\|_{1}\|B\| \\
& \|B A\| \leq\|A\|_{1}\|B\| \quad\left(A \in \sigma_{1}(H), B \in L(H, H)\right)
\end{aligned}
$$

and

$$
\left\|D_{x}^{(k)} G_{0}(x-y, y, t)\right\| \leq c t^{\frac{n+k}{2}} \exp \left(-c \frac{|x-y|^{2}}{t}\right), \quad(k=0,1,2)
$$

which were proved in $[9,10]$ respectively and we shall suppose $t \in(0,1)$.

\section{The spectrum of operator $L$ asymptotic for- mula for the number of its eigenvalues}

In this section, the spectrum of operator $L$ is studied and an asymptotic formula is found for the number of eigenvalues which is less then $\lambda$, where $\lambda$ is a positive variable. First of all, to solve integral equation (1.8), we need to estimate the operator function $K(x, y, t)$. 
Theorem 3.1. If the conditions 1), 4) are satisfied and for every $t>0, x \in \mathbb{R}^{n} e^{-t Q(x)} \in \sigma_{1}(H)$ then

$$
\|K(x, y, t)\|_{1} \leq \text { const. } t^{-b}\|\exp (-c t Q(y))\|_{1} \exp \left[-c \frac{|x-y|^{2}}{t}\right],
$$

where $b=\max \left\{\frac{n+1}{2}, \frac{n-1}{2}+a\right\}$.

Proof. From the relations (1.4), (1.6), (2.1) and the functions $a_{i j}(x)$ satisfy the condition 1$)$, for $|x-y| \leq \frac{1}{2}$ we obtain

$$
\begin{aligned}
{\left[\frac{\partial}{\partial t}+L_{0}\left(x, \frac{\partial}{\partial x}\right)\right] G_{1}(x-} & \left.y, y, t)=\| L_{0}\left(x, \frac{\partial}{\partial x}\right)-L_{0}\left(y, \frac{\partial}{\partial x}\right)\right] G_{0}(x-y, y, t) \| \\
& \left.=\| \sum_{i, j=1}^{n}\left[a_{i j}(x)\right)-a_{i j}(y)\right] \frac{\partial^{2} G_{0}(x-y, y, t)}{\partial x_{i} \partial x_{j}} \| \\
& \leq \text { const.t } t^{-\frac{n+2}{2}}|x-y| \exp \left[-c \frac{|x-y|^{2}}{t}\right] \\
(3.1) \quad & \leq \text { const.t }{ }^{-\frac{n+2}{2}} \exp \left[-\frac{c}{2} \frac{|x-y|^{2}}{t}\right]
\end{aligned}
$$

If we use again (1.4) and (2.1), for $\frac{1}{2}<|x-y| \leq 1$ we find

$$
\begin{aligned}
\left.\| L_{0}\left(x, \frac{\partial}{\partial x}\right) G_{1}(x-y, y, t)\right) \| \leq & \text { const.t } t^{-\frac{n+2}{2}} \exp \left[-c \frac{|x-y|^{2}}{t}\right], \\
\left.\| \frac{\partial}{\partial t} G_{1}(x-y, y, t)\right) \| \quad & =|R(x, y)|\left\|\frac{\partial}{\partial t} G_{0}(x-y, y, t)\right\| \\
= & |R(x, y)|\left\|L_{0}\left(y, \frac{\partial}{\partial x}\right) G_{0}(x-y, y, t)\right\| \\
\leq & \text { const.t. } t^{-\frac{n+2}{2}} \exp \left[-c \frac{|x-y|^{2}}{t}\right]
\end{aligned}
$$

$$
\left\|\left[\frac{\partial}{\partial t}+L_{0}\left(x, \frac{\partial}{\partial x}\right)\right] G_{1}(x-y, y, t)\right\| \leq \text { const.exp }\left[-\frac{c}{2} \frac{|x-y|^{2}}{t}\right] .
$$

On the other hand, recall for $|x-y|>1 G_{1}(x-y, y, t) \equiv 0$ and from (3.1) and (3.2) we obtain

$$
\left\|\left[\frac{\partial}{\partial t}+L_{0}\left(x, \frac{\partial}{\partial x}\right)\right] G_{1}(x-y, y, t)\right\| \leq \text { const. } t^{-\frac{n+1}{2}} \exp \left[-\frac{c}{2} \frac{|x-y|^{2}}{t}\right] .
$$


By the hypothesis, since for $t>0, x \in \mathbb{R}^{n} e^{-t Q(x)} \in \sigma_{1}(H)$ we write

$\left.\|(Q(y)-Q(x)) e^{-t Q(y)} G_{1}(x-y, y, t)\right) \|_{1} \leq$ const. $\|(Q(y)-Q(x))$.

$$
e^{-\frac{t}{2} Q(y)}\|\| e^{-\frac{t}{2} Q(y)} \|_{1} t^{-\frac{n}{2}} \exp \left[-c \frac{|x-y|^{2}}{t}\right] .
$$

Since $Q(x)$ satisfies the condition 4$)$ we have

$$
\begin{aligned}
\left\|(Q(y)-Q(x)) e^{-\frac{t}{2} Q(y)}\right\| & \leq\left\|(Q(y)-Q(x)) Q^{-a}(y)\right\|\left\|Q^{a}(y) e^{-\frac{t}{2} Q(y)}\right\| \\
& \leq B|x-y| t^{-a}|| t^{a} Q^{a}(y) e^{-\frac{t}{2} Q(y)} \| \\
& \leq \text { const.t }{ }^{-a}|x-y| .
\end{aligned}
$$

If we consider this last relation for (3.4), then we obtain

$$
\begin{gathered}
\left.\|(Q(y)-Q(x)) e^{-t Q(y)} G_{1}(x-y, y, t)\right) \|_{1} \leq \text { const.t }^{-\frac{n-1}{2}-a} . \\
\left\|e^{-\frac{t}{2} Q(y)}\right\|_{1} \exp \left[-\frac{c}{2} \frac{|x-y|^{2}}{t}\right] .
\end{gathered}
$$

Since the derivatives of second order of $R(x)$ are continuous, from (1.4) and (2.1) we find

$$
\begin{gathered}
\left.\| e^{-t Q(y)} L_{1}\left(x, \frac{\partial}{\partial x}\right) G_{1}(x-y, y, t)\right) \|_{1} \\
\leq \text { const.t } t^{-\frac{n+1}{2}} \cdot\left\|e^{-t Q(y)}\right\|_{1} \exp \left[-c \frac{|x-y|^{2}}{t}\right] .
\end{gathered}
$$

From (1.9),(3.3),(3.5) and (3.6) we obtain

$$
\|K(x, y, t)\|_{1} \leq \leq \text { const. } t^{-b}\|\exp (-c t Q(y))\|_{1} \exp \left[-c \frac{|x-y|^{2}}{t}\right] .
$$


This proves theorem.

In a similar form of the proof of this theorem, the inequality

$$
\|K(x, y, t)\| \leq \text { const. } t^{-b} \exp \left[-c \frac{|x-y|^{2}}{t}\right]
$$

can be shown.

From theorem 3.1, (3.7) and the inequalities

$$
\begin{gathered}
\int_{-\infty}^{\infty} \exp \left[-c \frac{(\xi-z)^{2}}{t-\tau}-c \frac{(z-\eta)^{2}}{\tau}\right][(t-\tau) \tau]^{\frac{1}{2}} d z \\
\leq c(\epsilon) t^{\frac{1}{2}} \exp \left[-(c-\epsilon) \frac{(\xi-\eta)^{2}}{t}\right], \\
\frac{(\xi-z)^{2}}{t-\tau}+\frac{(z-\eta)^{2}}{t} \geq \frac{(\xi-\eta)^{2}}{t}
\end{gathered}
$$

which were proved in [10], it can be proved that the series

$$
\varphi(x, y, t)=\sum_{m=1}^{\infty} K_{m}(x, y, t)
$$

which is a solution of the equation (1.8), is convergent with respect to the norm in $\sigma_{1}(H)$ of such that $Q^{-l}(x) \in \sigma_{1}(H)$ for every $x \in \mathbb{R}^{n}$ and the inequalities

$$
\begin{gathered}
\|\varphi(x, y, t)\|_{1} \leq \text { const.exp }\left[-c \frac{|x-y|^{2}}{t}\right] \\
\left.\left\|Q^{-l}(y)\right\|_{1}+t^{-b}\left\|\exp \left(-c t Q^{-l}(y)\right)\right\|_{1}\right\}(3.9)
\end{gathered}
$$

$$
\|\varphi(x, y, t)\|_{1} \leq \text { const. }\left\|Q^{-l}(y)\right\|_{1} \exp \left[-c \frac{|x-y|^{2}}{t}\right], \quad(|x-y|>1)
$$

$$
\|\varphi(x, y, t)\| \leq \text { const. } t^{-b} \exp \left[-c \frac{|x-y|^{2}}{t}\right] .
$$

are satisfied. 
Theorem 3.2. If the coefficients of differential expression (1.1) satisfy the conditions 1$), 3), 4), 6)$ and for every $x \in \mathbb{R}^{n} Q^{-l}(x) \in$ $\sigma_{1}(H)$, then while $t \rightarrow+0$

$$
\begin{gathered}
G(x, y, t)=G_{1}(x-y, y, t) e^{-t Q(y)}+t^{1-b} \exp \left[-c \frac{|x-y|^{2}}{t}\right] \cdot\left\{\left\|Q^{-l}(y)\right\|_{1}+\right. \\
+\| \exp \left(-c t Q(y) \|_{1}\right\} O(1)
\end{gathered}
$$

where $O(1)$ is an operator function which is bounded with respect to the norm $\sigma_{1}(H)$ in variables $x, y$ and $t$ and $b=\max \left\{\frac{n+1}{2}, \frac{n-1}{2}+a\right\}$.

Proof. From (1.3), (1.4), (1.5) and (2.1) we obtain

$$
\begin{gathered}
\left\|G(x, y, t)-e^{t Q(y)} G_{1}(x-y, y, t)\right\|_{1} \leq \\
\leq A_{1} \int_{0}^{t}(t-\tau)^{-\frac{n}{2}} d \tau \int_{\mathbb{R}^{n}} \exp \left[-c \frac{|x-\xi|^{2}}{t-\tau}\right] \\
\|\exp [-(t-\tau) Q(\xi)] \varphi(\xi, y, \tau)\|_{1} d \xi \\
=A_{1} \int_{0}^{t}(t-\tau)^{-\frac{n}{2}} d \tau \int_{|y-\xi| \leq 1} \exp \left[-c \frac{|x-\xi|^{2}}{t-\tau}\right] \| \\
\left.\exp [-(t-\tau) Q(\xi)] \varphi(\xi, y, \tau)\right|_{1} d \xi \\
+A_{1} \int_{0}^{t}(t-\tau)^{-\frac{n}{2}} d \tau \int_{|y-\xi|>1} \exp \left[-c \frac{|x-\xi|^{2}}{t-\tau}\right] \| \\
\exp [-(t-\tau) Q(\xi)] \varphi(\xi, y, \tau) \|_{1} d \xi \\
=B_{1}+B_{2}
\end{gathered}
$$

where $A_{1}$ is a positive constant. By using the inequalities (3.9) and (3.11) we shall estimate the integral $B_{1}$ :

$$
\begin{gathered}
B_{1}=A_{1} \int_{0}^{t}(t-\tau)^{-\frac{n}{2}} d \tau \int_{|y-\xi| \leq 1} \exp \left[-c \frac{|x-\xi|^{2}}{t-\tau}\right] \cdot \| \\
\exp [-(t-\tau) Q(\xi)] \varphi(\xi, y, \tau) \mid{ }_{1} d \xi \\
\leq \text { const. } \int_{0}^{\frac{t}{2}}(t-\tau)^{-\frac{n}{2}} \tau^{-b} d \tau \int_{|y-\xi| \leq 1} \exp \left[-c \frac{|x-\xi|^{2}}{t-\tau}\right]\|\exp [-(t-\tau) Q(\xi)]\|_{1} .
\end{gathered}
$$




$$
\exp \left[-c \frac{|\xi-y|^{2}}{\tau}\right] d \xi+s a b i t \int_{\frac{t}{2}}^{t}(t-\tau)^{-\frac{n}{2}} d \tau \int_{|y-\xi| \leq 1} \exp \left[-c \frac{|x-\xi|^{2}}{t-\tau}\right] .
$$

$\exp \left[-(t-\tau) \alpha_{1}(\xi)\right] \exp \left[-c \frac{|\xi-y|^{2}}{\tau}\right]\left\{\tau^{-b}\|\exp [-c \tau Q(y)]\|_{1}+\left\|Q^{-l}(y)\right\|_{1}\right\} d \xi$

$$
=B_{1}^{(1)}+B_{1}^{(2)} .
$$

Since $\mathrm{Q}(\mathrm{x})$ satisfies the condition 6$)$ and from the inequality (3.8) and the formula

$$
\int_{0}^{t} \tau^{\alpha}(t-\tau)^{\beta} d \tau=t^{\alpha+\beta+1} B(\alpha+1, \beta+1) \quad(\alpha>-1, \beta>-1),
$$

where

$$
B(m, k)=\frac{\Gamma(m) \Gamma(k)}{\Gamma(m+k)}, \Gamma(m)=\int_{0}^{\infty} x^{m-1} e^{-x} d x
$$

for $B_{1}^{(1)}$ we find

$$
\begin{gathered}
B_{1}^{(1)}=\text { const. } \int_{0}^{\frac{t}{2}}(t-\tau)^{-\frac{n}{2}} \tau^{-b} d \tau \int_{|y-\xi| \leq 1} \exp \left[-c \frac{|x-\xi|^{2}}{t-\tau}\right] . \\
\|\exp [-(t-\tau) Q(\xi)]\|_{1} \exp \left[-c \frac{|\xi-y|^{2}}{\tau}\right] d \xi \\
\leq \text { const. }\left\|\exp \left[-c_{1} t Q(y)\right]\right\|_{1} \int_{0}^{\frac{t}{2}} \tau^{\frac{n}{2}-b} d \tau . \\
\int_{\mathbb{R}^{n}} \exp \left[-c \frac{|x-\xi|^{2}}{t-\tau}-c \frac{|\xi-y|^{2}}{\tau}\right][\tau(t-\tau)]^{-\frac{n}{2}} d \xi \\
\leq \text { const. } t^{1-b}\left\|\exp \left[-c_{1} t Q(y)\right]\right\|_{1} \exp \left[-c_{1} \frac{|x-y|^{2}}{t}\right] .
\end{gathered}
$$

In the similar form, we can estimate $B_{1}^{(2)}$ :

$$
B_{1}^{(2)}=\text { const. } \int_{\frac{t}{2}}^{t}(t-\tau)^{-\frac{n}{2}} d \tau \int_{|y-\xi| \leq 1} \exp \left[-c \frac{|x-\xi|^{2}}{t-\tau}\right] \exp \left[-(t-\tau) \alpha_{1}(\xi)\right]
$$




$$
\begin{aligned}
& \quad \exp \left[-c \frac{|x-\xi|^{2}}{t-\tau}-c \frac{|\xi-y|^{2}}{\tau}\right]\left\{\tau^{-b}\|\exp [-c \tau Q(y)]\|_{1}+\left\|Q^{-l}(y)\right\|_{1}\right\} d \xi \\
& \leq \text { const. }\left\{t^{-b}\left\|\exp \left[-c_{1} t Q(y)\right]\right\|_{1}+\left\|Q^{-l}(y)\right\|_{1}\right\} \cdot \\
& \quad \int_{0}^{t} \tau^{\frac{n}{2}} d \tau \int_{\mathbb{R}^{n}} \exp \left[-c \frac{|x-\xi|^{2}}{t-\tau}-c \frac{|\xi-y|^{2}}{\tau}\right][\tau(t-\tau)]^{-\frac{n}{2}} d \xi \\
& \leq \text { const.t }\left\{t^{-b}\left\|\exp \left[-c_{1} t Q(y)\right]\right\|_{1}+\left.\left\|Q^{-l}(y)\right\|\right|_{1}\right\} \exp \left[-c_{1} \frac{|x-y|^{2}}{t}\right]
\end{aligned}
$$

From (3.13), (3.14) and the last relation we find

$B_{1} \leq$ const.t $\left\{t^{-b}\left\|\exp \left[-c_{1} t Q(y)\right]\right\|_{1}+\left\|Q^{-l}(y)\right\|_{1}\right\} \exp \left[-c_{1} \frac{|x-y|^{2}}{t}\right] \leq$

$$
\leq \text { const. } t^{1-b} \exp \left[-c_{1} \frac{|x-y|^{2}}{t}\right]\left\{\left\|\exp \left[-c_{1} t Q(y)\right]\right\|_{1}+\left\|Q^{-l}(y)\right\|_{1}\right\} .
$$

This time, let us estimate the expression $B_{2}$ in (3.12) using the inequalities (3.8) and (3.10).

$$
\begin{gathered}
B_{2}=A_{1} \int_{0}^{t}(t-\tau)^{-\frac{n}{2}} d \tau \int_{|y-\xi|>1} \exp \left[-c \frac{|x-\xi|^{2}}{t-\tau}\right] \\
\|\exp [-(t-\tau) Q(\xi)] \varphi(\xi, y, \tau)\|_{1} d \xi \\
\leq A_{1} \int_{0}^{t}(t-\tau)^{-\frac{n}{2}} d \tau \int_{|y-\xi|>1}|| \exp [-(t-\tau) Q(\xi)] \| \\
\exp \left[-c \frac{|x-\xi|^{2}}{t-\tau}\right]\|\varphi(\xi, y, \tau)\|_{1} d \xi \\
\leq \operatorname{const} \cdot \| Q^{-l}(y)||_{1} \int_{0}^{t} \tau^{\frac{n}{2}} d \tau \int_{\mathbb{R}^{n}} \\
\exp \left[-c \frac{|x-\xi|^{2}}{t-\tau}-c \frac{|\xi-y|^{2}}{\tau}\right][\tau(t-\tau)]^{-\frac{n}{2}} d \xi \\
\leq \operatorname{const.t}|| Q^{-l}(y) \mid{ }_{1} \exp \left[-c_{1} \frac{|x-y|^{2}}{t}\right] .
\end{gathered}
$$


From (3.12), (3.15) and (3.16) we obtain

$$
\begin{gathered}
\left\|G(x, y, t)-e^{-t Q(y)} G_{1}(x-y, y, t)\right\|_{1} \leq \\
\leq \text { const.t }{ }^{1-b} \exp \left[-c_{1} \frac{|x-y|^{2}}{t}\right]\left\{\left\|\exp \left[-c_{1} t Q(y)\right]\right\|_{1}+\left\|Q^{-l}(y)\right\|_{1}\right\}
\end{gathered}
$$

or

$$
\begin{gathered}
G(x, y, t)=G_{1}(x-y, y, t) e^{-t Q(y)}+t^{1-b} \exp \left[-c_{1} \frac{|x-y|^{2}}{t}\right] . \\
\left\{\left\|\exp \left[-c_{1} t Q(y)\right]\right\|_{1}+\left\|Q^{-l}(y)\right\|_{1}\right\} O(1) .
\end{gathered}
$$

This completes the proof.

Now, we can prove that the spectrum of $L$ is pure discrete and find an asymptotic formula for the number of its eigenvalues.

Theorem 3.3. If the coefficients of differential expression (1.1) satisfy the conditions 1) -7) then the spectrum of $L$ is pure discrete.

Proof. The operator function $G_{0}(x-y, y, t)$ is in the form

$$
G_{0}(x-y, y, t)=\frac{I}{(2 \pi)^{n}} \int_{\mathbb{R}^{n}} \exp \left[-t \sum_{i, j=1}^{n} a_{i} j(y) s_{i} s_{j}+i(s, x-y)\right] d s,
$$

where $I: H \rightarrow H$ is an identity operator. By translating $s_{i}=t^{-\frac{1}{2}} \xi_{i}$ we have

$G_{0}(x-y, y, t)=\frac{I}{(2 \pi \sqrt{t})^{n}} \int_{\mathbb{R}^{n}} \exp \left[-\Sigma_{i, j=1}^{n} a_{i} j(y) \xi_{i} \xi_{j}+i t^{-\frac{1}{2}}(\xi, x-y)\right] d \xi$.

From here, we find

$$
G_{0}(0, x, t)=\frac{I}{(2 \pi \sqrt{t})^{n}} \int_{\mathbb{R}^{n}} \exp \left[-\sum_{i, j=1}^{n} a_{i} j(x) \xi_{i} \xi_{j}\right] d \xi=\frac{\Phi(x)}{(2 \pi \sqrt{t})^{n}} I .
$$

From the formulas (1.4), (3.17) and theorem 3.2 we obtain

$$
\operatorname{tr} G(x, x, t)=\frac{\Phi(x)}{(2 \pi \sqrt{t})^{n}} \operatorname{tr} e^{-t Q(x)}+O(1) t^{1-b}\left[\left\|e^{-c t Q(x)}\right\|_{1}+\left\|Q^{-l}(x)\right\|_{1}\right] .
$$


By hypothesis, for every $x \in \mathbb{R}^{n}, Q(x) \geq I$ and hence

$$
\left\|e^{-c t Q(x)}\right\|_{1}=\operatorname{tr} e^{-c t Q(x)} .
$$

On the other hand, since $\mathrm{Q}(\mathrm{x})$ satisfies the conditions 5) -7) and from (3.18) we find

$$
\int_{\mathbb{R}^{n}} \operatorname{tr} G(x, x, t) d x=\frac{1}{(2 \pi \sqrt{t})^{n}} \int_{\mathbb{R}^{n}} \Phi(x) \operatorname{tre}^{-t Q(x)} d x+O(1) t^{1-b} .
$$

$$
\int_{\mathbb{R}^{n}} \Phi(x) \operatorname{tr} e^{-t Q(x)} d x+O(1) t^{1-b}
$$

Since the expression $\sum_{i, j=1}^{n} a_{i} j(x) \xi_{i} \xi_{j}$ satisfies the condition 2) there exist positive constants $M_{1}$ and $M_{2}$ such that

$$
M_{1}<\Phi(x)<M_{2}
$$

Thus, from (3.19) we obtain

$$
\int_{\mathbb{R}^{n}} \operatorname{tr} G(x, x, t) d x<\infty .
$$

Since $u=e^{-t L} \psi(x)$ and $u=\int_{\mathbb{R}^{n}} G(x, y, t) \psi(y) d y, G(x, y, t)$ is the kernel of positive bounded operator $e^{-t L}(t>0)$. In this case, for every $t>0, e^{-t L}$ is the kernel operator, [8]. For the spectrums of operators $e^{-t L}$ and $L$

$$
s\left\{e^{-t L}\right\}=e^{-t s\{L\}} .
$$

Since for every $t>0, e^{-t L}$ is a complete continuous operator and from the last formula we obtain that the spectrum of $L$ is pure discrete.

Let $\lambda_{1} \leq \lambda_{2} \leq \ldots \leq \lambda_{n} \leq \ldots$, be eigenvalues of $L$ and let

$$
N(\lambda)=\Sigma_{\lambda_{n}<\lambda} 1
$$

where $\lambda>0$.

Theorem 3.4. If the coefficients of differential expression (1.1) satisfy the conditions 1)-8) then while $\lambda \rightarrow \infty$ the asymptotic formula

$$
N(\lambda) \sim \frac{1}{(2 \pi)^{n} \Gamma\left(\frac{n+2}{2}\right)} \Sigma_{i} \int_{\alpha_{i}(x)<\lambda} \Phi(x)\left(\lambda-\alpha_{i}(x)\right)^{\frac{n}{2}} d x
$$


is satisfied.

Proof. The following equality holds:

$$
t r e^{-t L}=\int_{0}^{\infty} e^{-\lambda t} d N(\lambda)
$$

From (3.19) and (3.20) while $t \rightarrow+0$ we obtain the asymptotic formula

$$
\int_{\mathbb{R}^{n}} \operatorname{tr} G(x, x, t) d x=F(t)+o(1) F(t),
$$

where

$$
F(t)=\frac{1}{(2 \pi \sqrt{t})^{n}} \int_{\mathbb{R}^{n}} \Phi(x) t r e^{-t Q(x)} d x
$$

and $o(1)$ is a function of $t$ satisfying the condition $\lim _{t \rightarrow+0} o(1)=0$.

On the other hand, in [8] it is given

$$
t r e^{-t L}=\int_{\mathbb{R}^{n}} \operatorname{tr} G(x, x, t) d x .
$$

From this last, (3.21) and (3.22) we find

$$
\int_{0}^{\infty} e^{-\lambda t} d N(\lambda)=\int_{\mathbb{R}^{n}} \operatorname{tr} G(x, x, t) d x=F(t)+o(1) F(t) .
$$

Moreover,

$$
\begin{gathered}
F(t)=\frac{1}{(2 \pi \sqrt{t})^{n}} \int_{\mathbb{R}^{n}} \Phi(x)\left[\Sigma_{i=1}^{\infty} e^{-t \alpha_{i}(x)}\right] d x \\
=\frac{1}{(2 \pi \sqrt{t})^{n}} \Sigma_{i=1}^{\infty} \int_{\mathbb{R}^{n}} \Phi(x) e^{-t \alpha_{i}(x)} d x .
\end{gathered}
$$

On the other hand, in [11] it is shown that

$$
\frac{1}{(2 \pi \sqrt{t})^{n}} \int_{\mathbb{R}^{n}} \Phi(x) e^{-t \alpha_{i}(x)} d x=\int_{0}^{\infty} e^{-\lambda t} d \rho_{i}(\lambda),
$$

where

$$
\rho_{i}(\lambda)=\frac{1}{(2 \pi)^{n} \Gamma\left(\frac{n+2}{2}\right)} \int_{\alpha_{i}(x)<\lambda} \Phi(x)\left(\lambda-\alpha_{i}(x)\right)^{\frac{n}{2}} d x .
$$


Hence, from the last three equalities we obtain

$$
F(t)=\int_{0}^{\infty} e^{-\lambda t} d \rho(\lambda)
$$

where

$$
\rho(\lambda)=\frac{1}{(2 \pi)^{n} \Gamma\left(\frac{n+2}{2}\right)} \Sigma_{i} \int_{\alpha_{i}(x)<\lambda} \Phi(x)\left(\lambda-\alpha_{i}(x)\right)^{\frac{n}{2}} d x .
$$

From (3.24) and (3.25), while $t \rightarrow+0$ we find

$$
\int_{0}^{\infty} e^{-\lambda t} d N(\lambda) \sim \int_{0}^{\infty} e^{-\lambda t} d \rho(\lambda)
$$

By hypothesis, we have

$$
\lambda \rho^{\prime}(\lambda)<a_{0} \rho(\lambda)
$$

Thus, from (3.26) and according to [12], while $\lambda \rightarrow \infty$ we obtain

$$
N(\lambda) \sim \rho(\lambda)
$$

or

$$
N(\lambda) \sim \frac{1}{(2 \pi)^{n} \Gamma\left(\frac{n+2}{2}\right)} \Sigma_{i} \int_{\alpha_{i}(x)<\lambda} \Phi(x)\left(\lambda-\alpha_{i}(x)\right)^{\frac{n}{2}} d x
$$

Example 3.1. Here we will give an example of scaler functions $a_{i j}(x)(i, j=1,2, . ., n)$ and an operator function $Q(x)$ satisfying the conditions 1) -8$)$. Now, let

$$
a_{i j}(x)=\left\{\begin{array}{lll}
1 & , & i=j \\
0 & , & i \neq j
\end{array} \quad(i, j=1,2,3, \ldots, n)\right.
$$

These functions obviously satisfy the conditions 1) - 2).

For an orthonormal basis $\left\{e_{i}\right\}_{i=1}^{\infty}$ of separable Hilbert space $H$, we define the operator function in the form

$$
Q(x) f=\Sigma_{i=1}^{\infty}\left(f, e_{i}\right) i^{4}\left(1+|x|^{5 / 4}\right) e_{i} \quad, \quad(\mathrm{f} \in \mathrm{D}(\mathrm{Q}(\mathrm{x})))
$$

It can be easily shown that the operator function $Q(x)$ satisfies the conditions 3) -8). 


\section{References}

[1] Kostyuchenko, A. G., and Levitan, B. M., "Asymptotic behavior of the eigenvalues of the Sturm-Liouville operator problem", Funct. Anal. Appl. 1, pp. 75-83, (1967).

[2] Gorbauuk, M.L., " Self adjoint boundary value problems of second order differential equation with unbounded operator coefficients", Funks. analiz i yego pril., 5, No:1, pp. 10-21, (1971).

[3] Gorbacuk, V.I., Gorbacuk, M.L., "On a class boundary value problems for Sturm-Liouville equation with operator coefficients", Ukr. mat. jur., T.24, No:3, pp. 291-305, (1972).

[4] Gorbacuk, V. I., Gorbacuk, M. L., " Some problems of spectral theory of differential equations in elliptic type in space of vector functions", Ukr. mat. jur., T.28, No:3, pp. 313-324, (1976).

[5] Otelbayev, M., "On Titcmars method of restriction of resolvent", Dokl. A.N. SSSR, T.281, No:4, pp. 787-790, (1973).

[6] Solomyak, M. Z., "Asymptotics of the spectrum of the Schrodinger operator with non-regular homogeneous potential", Math. USSR Sbornik, Vol. 55, No:1, pp. 19-37, (1986).

[7] Maksudov, F. G., Bairamoglu, M., Adiguzelov,E. E., "On asymptotics of spectrum and trace of high order differential operator with operator coefficients", Doa Turkish Journal of mathematics, vol. 17, number 2, 1993, 113-128

[8] Kirillov, A. A., Elementary theory of representations, Springer verlag, New York, (1976).

[9] Cohberg,. C., and Krein, M. G., "Introduction to the theory of linear non-self adjoint operators", Translation of Mathematical Monographs volume 18, Amer. Math. Sos., Providence, R. I. (1969).

[10] Eydelman, S. D., "Parabolic Systems", North-Holland Publishing Company Amsterdam, (1969). 
[11] Kostyuchenko, A. G., "The asymptotic behavior of the spectral function of self adjoint operators in elliptic type", V kn "Cetvyontaya matem. skola" , Kiev, pp. 42-117, (1968).

[12] Korenblyum, B. I., "General Tauber Teorems for the ration of functions", DAN SSSR, 88 No:5, pp. 745-748, (1953).

Received : April 2000.

\section{E. Adiguzelov}

Yildiz Technical University

Department of Mathematics

Davutpasa Campus

34210 Eesenler

Istanbul

Turkey

E-mail : adiguzelov@yildiz.edu.tr

\section{H. Avci}

Yildiz Technical University

Department of Mathematics

Davutpasa Campus

34210 Esenler

Istanbul

Turkey

E-mail : avci@yildiz.edu.tr

and

\section{E. Gul}

Yildiz Technical University

Deppartment of Mathematics

Davutpasa Campus

34210 Esenler

Istanbul

Turkey

E-mail : gul@yildiz.edu.tr 